\title{
Müttersprache - Zu den Auswirkungen eines Sprachkurses für Mütter auf deren Babys
}

Der Umlaut beim ersten Vokal des Titels ist gewollt, denn es geht im Folgenden nicht um die von einem Menschen in der frühen Kindheit ohne jeglichen Unterricht erlernte Sprache, sondern um die Sprache von Müttern und Vätern, wenn sie mit ihren kleinen Kindern sprechen. Im Englischen spricht man auch von "child directed speech“, „motherese“ und - sachlich nicht ganz korrekt, politisch dafür aber umso mehr „parentese“. Zwar sprechen auch Väter mit ihren Kindern etwas anders, machen aber zwischen Erwachsenen und Kindern deutlich seltener einen Unterschied als Mütter. Und wenn überhaupt, dann ändern sie im Vergleich zu Müttern, ihre Art zu sprechen nur wenig. „Fatherese“ gab es daher in der psychologischen Literatur nie. ${ }^{1}$

\section{Babys hören schlechter als}

\section{Erwachsene}

Neugeborene hören nicht so gut wie Erwachsene, d. h. alles hört sich für sie etwas leiser an und ihre Hörschwellen (diese sind abhängig von der Tonfrequenz) sind erhöht ( Abb. 1).

Die Ursachen der - völlig normalen (!) - Hörminderung beim Säugling und Kleinkind sind vielfältig: Zum einen ist die Überleitung des Schalls in das Innenohr noch nicht so funktionsfähig wie beim Erwachsenen, der Gehörkanal ist kürzer, das Mittelohr hat ein kleineres Volumen und die Dichte und räumliche Orientierung der umgebenden Strukturen sind noch nicht vollständig entwickelt. „Aufgrund dieser anatomischen Veränderungen wird die Leitung der Schallenergie durch den Schallleitungsapparat mit zunehmendem Alter in der Kindheit effizienter“, beschreiben Werner

1 So ergab eine Suche nach "fatherese“ in PubMed am 4.3.2020 „0 results “ wohingegen eine Suche nach „motherese“ „55 results“ ergab. Sucht man bei Google, so findet man einige wenige Hinweise auf "fatherese“, die im Wesentlichen sagen, dass es das entweder gar nicht gibt oder dass es wegen politischer Korrektheit erwähnt wird. und Leibold [46] diesen Sachverhalt im Einzelnen. Das Innenohr hingegen ist bei Geburt vollständig entwickelt. ${ }^{2}$

Aber nicht nur die Energie akustischer Signale am Eingang des Innenohrs ist bei Babys geringer, sondern auch die Verarbeitung der elektrischen Signale vom Innenohr im Gehirn ist noch nicht voll entwickelt. Auf Hirnstammebene scheinen wichtige synaptische Verschaltungen zwischen 3 und 6 Monaten zu reifen, wie man aus elektrophysiologischen Befunden zu akustischen Hirnstammpotenzialen ableiten kann. Nach dem 7. Lebensmonat erscheinen diese Potenziale jedoch nahezu ausgereift (d.h. „wie beim Erwachsenen“). Die weitere spätere Entwicklung des Hörens geht damit am ehesten auf das Konto von Neuroplastizität, d. h. von verbesserter Leistungsfähigkeit des akustischen Analyseapparats durch seine Benutzung. So entsteht die räumliche Karte zur Lokalisation von Schallquellen bekanntermaßen über eine Kalibrierung mithilfe des Sehsystems, d. h. in jedem Falle erfahrungsabhängig. Babys und Kleinkinder sind entsprechend schlecht im Lokalisieren von bewegten Schallquellen ( $\triangleright$ Abb. 2 ).

Vor allem aber für das Hören im Alltag gilt, dass die Unterscheidung verschiedener Schallquellen - man spricht von „sound source segregation“ bzw. „autitory scene analysis“ - auf top-down gesteuerten Mechanismen beruht, also nur mit Hilfe von Vorerfahrungen funktioniert [4] ( Abb. 3). Die Fähigkeit von kleinen Kindern, akustische Signale im Hinblick auf ihre Bedeutung kortikal zu verarbeiten, entwickelt sich also deutlich langsamer als die „Mechanik" der akustischen Vorverarbeitung im Hirnstamm. Lassen wir noch einmal Werner und Leibold [46, S. 82] zu Worte

2 „Converging evidence indicates that neither the rapid improvements in absolute auditory sensitivity occurring in early infancy nor the later progressive improvements occurring into childhood can be accounted for by maturation of the inner ear" [46, S.69]. kommen: „Kleinkinder und Kinder lernen immer besser, eine bestimmte Klanggestalt von irrelevanten Hintergrundgeräuschen zu trennen. Wenn sie älter werden, werden sie empfindsamer gegenüber bestimmten Eigenschaften von Klanggestalten, die sie zuvor offenbar nicht zu bemerken schienen. Schließlich sind Kinder in der Lage, die bedeutsamsten Aspekte des Gehörten auszuwählen, und werden hierdurch zu effizienten Informationsverarbeitern.“3

\section{Was ist Müttersprache?}

Mütter scheinen zu spüren, dass das Hörvermögen des Säuglings noch nicht ganz optimal ausgebildet ist, denn man weiß schon lange, dass sie ihre Stimme beim Sprechen mit dem Neugeborenen systematisch verändern. Ausgiebige Studien der Arbeitsgruppe um Anne Fernald ergaben bereits in den 1980er-Jahren, dass Mütter anders sprechen, wenn sie mit ihren Babys kommunizieren ( $\mathbf{A b b} \mathbf{4}$ ): Die Tonhöhe (Grundfrequenz) nimmt zu, die Variation der Tonhöhe nimmt zu (stärker modulierte Sprachmelodie), die Amplitude der Töne (deren Lautheit) nimmt zu und die Länge der Pausen zwischen Wörtern nimmt zu, die Länge der Sätze nimmt hingegen ab. Zugleich sind die Strukturen der Sprachmelodie (der „Singsang der Sprache“) sehr einfach und sehr ähnlich.

Fernald konnte nicht nur nachweisen, dass Mütter mit Babys systematisch anders sprechen. Sie konnte auch (an 48 Babys im Alter von jeweils 4 Monaten) nachweisen, dass die Babys die Müttersprache der normalen Sprache vorziehen, d. h. sich lauteren, stärker modulierten und klarer strukturierten Äußerungen eher zuwenden als der norma-

3 „Infants and children progressively learn to separate a target sound from irrelevant sounds. As children grow older, they become sensitive to aspects of sounds that they previously appeared not to notice. Finally, children are able to choose the most informative aspects of sound to be efficient information processors.“ 


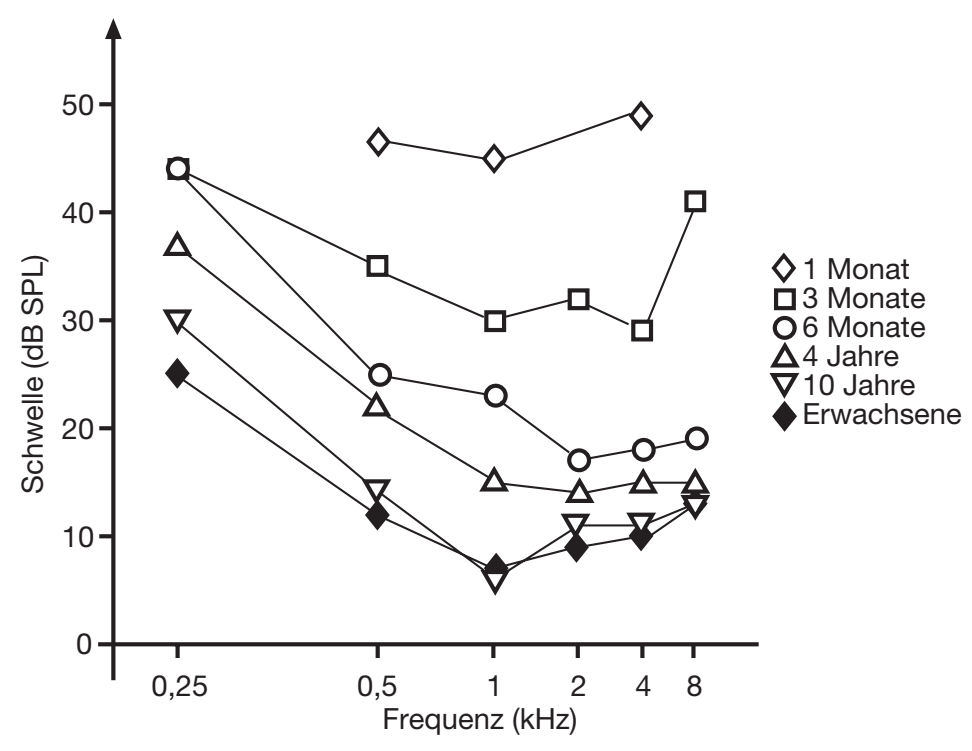

Abb. 1 Hörschwellen bei Kindern verschiedenen Alters und bei Erwachsenen in Abhängigkeit von der Tonhöhe. Die Hörschwellen für hohe Frequenzen verbessern sich bis zum 6. Lebensmonat rasch, wohingegen bei niedrigen Frequenzen noch bis in die mittlere Kindheit Hörschwellenverbesserungen auftreten (nach Daten aus [46]).

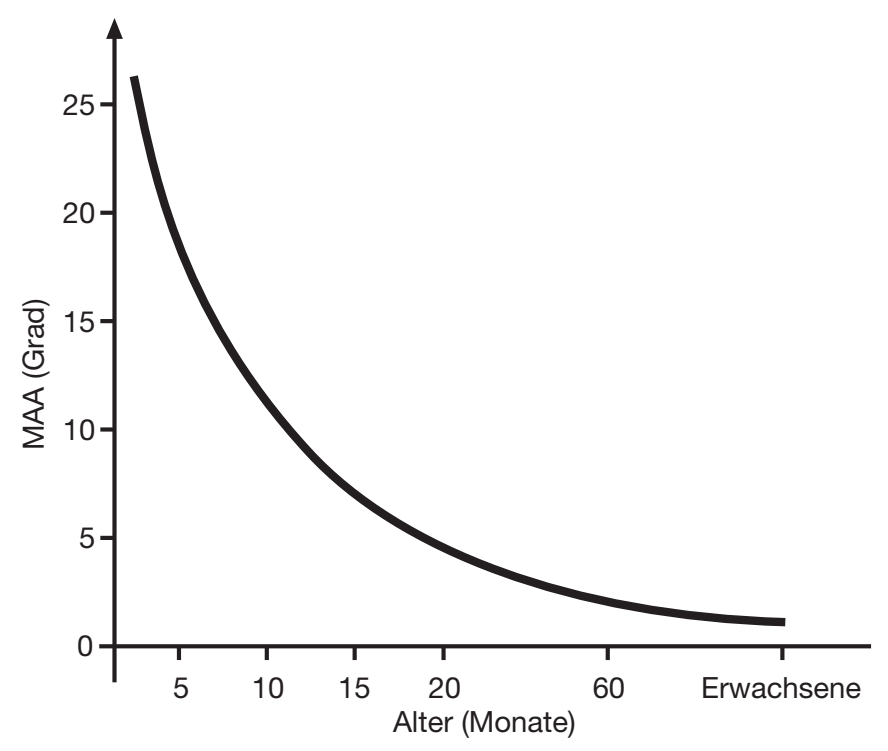

- Abb. 2 Entwicklung der Fähigkeit zur Lokalisierung einer sich horizontal bewegenden Schallquelle. Der kleinste hörbare Winkel (minimum audible angle, MAA) nimmt in den ersten 5 Lebensjahren ( 60 Monaten) deutlich ab und liegt bei manchen geübten Erwachsenen bei $1-2$ Grad.

len Sprache. Wahrscheinlich hilft eine solche veränderte Sprache den Säuglingen bei der Aufrechterhaltung der Aufmerksamkeit, vielleicht wird auch Wichtiges besser von Unwichtigem getrennt, und möglicherweise hat die höhere Stimme auch einen positiven affektiven Beiklang für das kleine Kind
$[13,14]$. Halten wir fest: Müttersprache erreicht die Babys besser als normale Sprache.

Sie fördert daher die Sprachentwicklung der Kinder, wie man mittlerweile ebenfalls empirisch nachweisen konnte. In einer methodisch sehr hochwertigen und im Fach- blatt Science publizierten Studie konnten Patricia Kuhl und Mitarbeiter [18] bei 30 Müttern aus den USA, Russland und Schweden zeigen, dass die Unterscheidbarkeit der Laute durch die Veränderungen beim Sprechen von Müttersprache zunimmt ( $\triangleright$ Abb.5). Sie untersuchten die Eigenschaften einzelner Phoneme, wenn die Mütter normal oder in „Müttersprache“ sprachen.

„Unsere Befunde zeigen, dass der Sprachinput von Kindern über die Kulturen hinweg universelle Eigenschaften besitzt, die dem Erlernen der Sprache förderlich sind. Diese Charakteristika werden wahrscheinlich vom sich entwickelnden Gehirn des Kindes genutzt“, kommentierten die Autoren [18, S. 686] ihre Ergebnisse. 5 Jahre später wurden - nochmals im Fachblatt Science - ähnliche Analysen zur in natürlichem Setting zuhause aufgenommener Sprache von Müttern mit ihren Babys und mit Erwachsenen mit sehr ähnlichen Ergebnissen publiziert: Anstatt von Müttersprache war von Hyperartikulation die Rede. Gemeint war damit die Vergrößerung des „Vokal-Dreiecks" auf der vom ersten und zweiten Formanten aufgespannten Frequenzebene ( Abb. 6). Die Ansprache an Haustiere lag dabei interessanterweise zwischen der an Erwachsene und der an Babys.

\section{Müttersprache kommt beim}

\section{Baby besser an}

Bereits im Jahr 2003 wurde erstmals in einer Studie aus China nachgewiesen, dass die Größe des Lautdreiecks bei den Müttern, mit der Fähigkeit zur Sprachwahrnehmung in einem entsprechenden Test ihrer Kinder korrelierte [23]. Diese Tests wurden im Alter von 6 bis 8 Monaten und dann nochmals im Alter von 10 bis 12 Monaten durchgeführt. In diesem Alter kann man Sprachverständnis nicht erfragen, sondern muss Verhaltensparadigmen verwenden. Man präsentierte hierzu gleiche Laute und trainierte die Kinder zuvor, bei einem Lautunterschied den Kopf zum Lautsprecher, aus dem die Laute kamen, zu wenden. Daraufhin erhielten sie einen belohnenden Reiz („a black box lit up and a mechanical bear pounded a drum “4). Man konnte damit die Unterscheidungsfä-

4 „Eine schwarze Kiste leuchtete hell auf und ein mechanischer Bär fing an zu trommeln.“ 
higkeit für verschiedene Wörter messen. Die gefundenen Zusammenhänge zwischen Deutlichkeit der Sprache der Mutter (Größe des Lautdreiecks) und der Sprachwahrnehmung der Kinder waren mit $r=0,7$ in beiden Altersgruppen hoch und durch andere Variablen (Bildungsstand oder soziökonomischer Status der Mütter) nicht zu erklären. 2 weitere Experimente an 48 Kindern im Alter von 21 Monaten und 16 Kindern im Alter von 27 Monaten zeigten darüber hinaus, dass mit 21 Monaten neue Wörter überhaupt nur dann gelernt wurden, wenn sie in Müttersprache gehört wurden. Im Alter von 27 Monaten hingegen lernen Kinder auch dann neue Wörter, wenn sie diese in ganz normaler „Erwachsenensprache“ hören [25].

Eine Studie an 26 Kindern, deren Sprachinput im Alter von 11 bis 14 Monaten mit einem kleinen Rekorder aufgezeichnet und auf den Sprachstil analysiert wurde, fand eine erhöhte Sprachfähigkeit im Alter von 24 Monaten (höhere Rate an gesprochenen Wörtern) bei Kindern, die zuvor mehr Müttersprache gehört hatten [31]. Eine weitere Untersuchung der Kinder zu einem späteren Zeitpunkt ergab, dass die Mütter während des 3. Lebensjahres zunehmend auf Müttersprache verzichteten und mit ihren Kindern „normal“ sprachen. Der Effekt der Verwendung von Müttersprache, der sich bei den 1-Jährigen zeigte, war auch im Alter von 33 Monaten noch nachzuweisen [32].

\section{Coaching zum Lernen von Müttersprache}

Aufgrund der genannten Erkenntnisse ging man in der Arbeitsgruppe um Patricia Kuhl den nächsten logischen Schritt und untersuchte den Einfluss von einem Training der Eltern in Müttersprache auf die Sprachentwicklung der Kinder [9]. In einer randomisierten kontrollierten Studie wurden Familien mit einem 6 Monate alten gesunden Kind entweder einer Kontrollgruppe ( $n=24)$ oder einer von 2 Interventionsgruppen zugeordnet. Die Intervention bestand im Coaching der Eltern und beinhaltete professionelles Feedback zum sprachlichen Umgang der Eltern mit ihrem Kind $(n=53)$. Das Coaching fand etwa alle 3 Wochen für jede teilnehmende Familie einzeln statt - meistens waren nur die Mütter dabei, manchmal auch die Väter und selten nur die Väter.

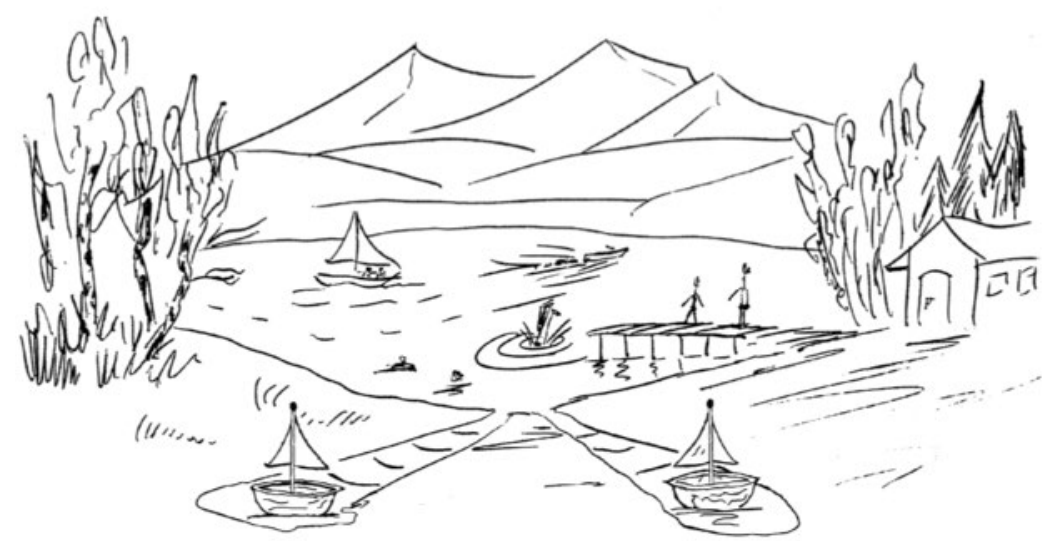

- Abb. 3 Gedankenexperiment (nach Daten aus [4, S. 5f]) zur Funktion des Gehörs. Stellen Sie sich vor, Sie sitzen mit dem Rücken zur Landschaft genau zwischen den beiden kleinen „Kanälen“, an deren Ende die kleinen Nussschalen-Segelbote schwimmen. Sie können nur das Auf und Ab der Segel beobachten. Daraus sollen Sie dann erschließen, was auf dem See los ist. - Etwa so geht es unserem Gehörsinn, denn er verarbeitet ja nichts weiter als das bisschen "Gewackel“ von linkem und rechtem Trommelfell. Daraus - und nur daraus (!) - entsteht für einen geübten Hörer die ganze räumliche Akustik, mit unterschiedlichen Schallquellen an unterschiedlichen Orten unterschiedlicher Lautstärke etc. Das Problem der akustischen Informationsverarbeitung - analog zum Rückschluss von der Bewegung der Segel beider Schiffchen auf die Aktivitäten auf dem See - wird so überhaupt erst deutlich [41, S.47].
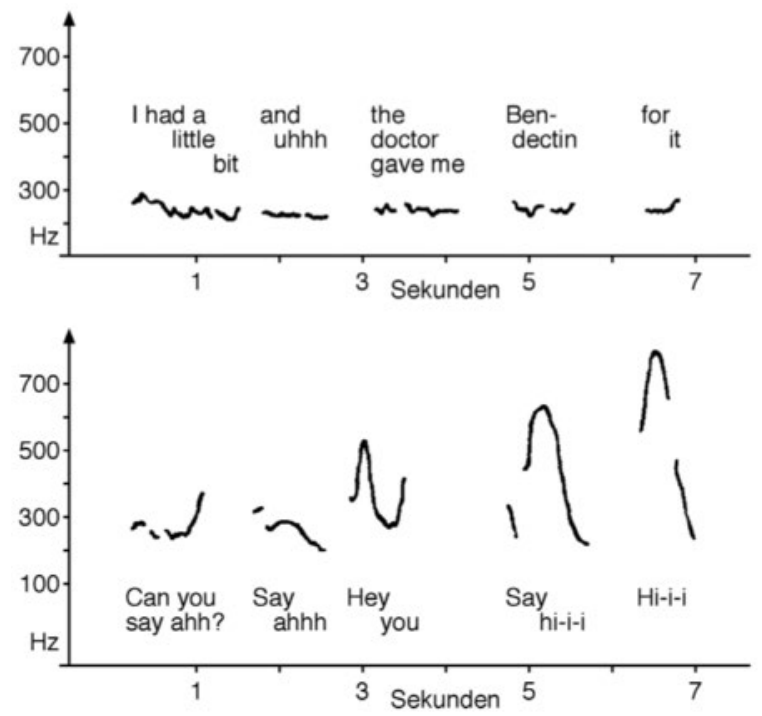

- Abb.4 Intonationskonturen (Grundfrequenz F0) über die Zeit (in Sekunden) der Stimme einer Frau zu einem anderen Erwachsenen (oben) und zu einem 4 Monate alten Säugling (nach Daten aus [11, S. 187]). Man erkennt vor allem die deutlich größere Variabilität der Grundfrequenz und kann die längeren Pausen ausmachen. Stellt man sich das Ganze zudem auch noch lauter vor (auf dem Bild nicht ersichtlich), dann wird klar, dass Mütter mit Babys anders sprechen, eben „Müttersprache“.

Vor Beginn der Studie wurden die Familien einzeln besucht und mit ihnen der Zweck der Studie, die Bedeutung der an das Kind gerichteten Besonderheiten der Müttersprache und deren einzelne Charakteristi- ka besprochen. Auch wurde den Eltern der kleine tragbare Sprachrekorder für die Kinder gezeigt und dessen Funktion und Handhabung erklärt. Hierdurch wurden im Alter von 6 Monaten (Ausgangswert), 10 Mona- 

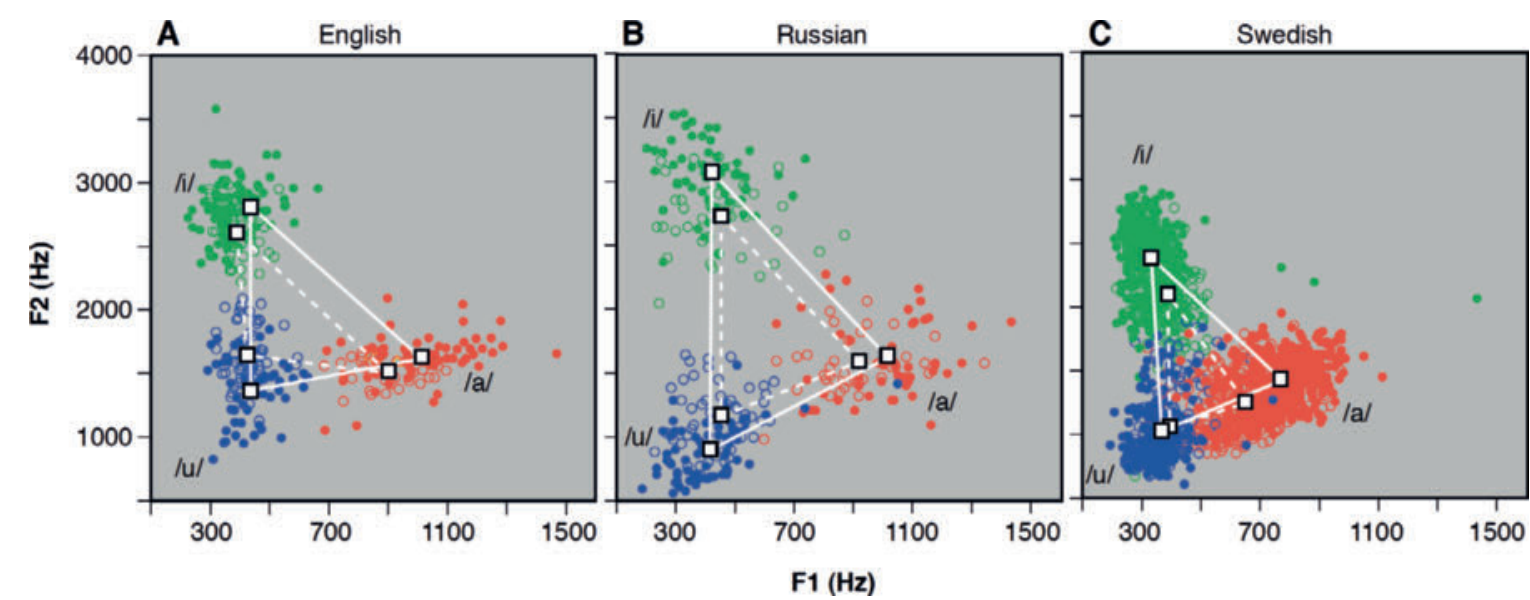

- Abb. 5 Jeder Punkt in den 3 Abbildungen bezieht sich auf eine Messung. Man verwendete Äußerungen von Müttern gegenüber Babys (gefüllte Kreise) und gegenüber Erwachsenen (offene Kreise) und untersuchte die Vokale „““ (grün), „a“ (rot) und „““ (blau) in 3 Sprachen: Englisch, Russisch und Schwedisch. Gemessen wurden die Grundfrequenzen der ersten beiden Sprachformanten, wobei es sich um Frequenzbänder handelt, die man aus Untersuchungen zur Sprache gut kennt [41]. Die Zentren der Punktwolken („center of gravity“) wurden mit Linien verbunden (normale Sprache: gestrichelte Linie; Müttersprache: durchgezogene Linie), wodurch sehr schön deutlich wird, dass die Unterscheidbarkeit der Laute bei Müttersprache höher ist, denn sie liegen in der von den Formaten aufgespannten Ebene weiter auseinander (nach Daten aus [18, S. 685]).

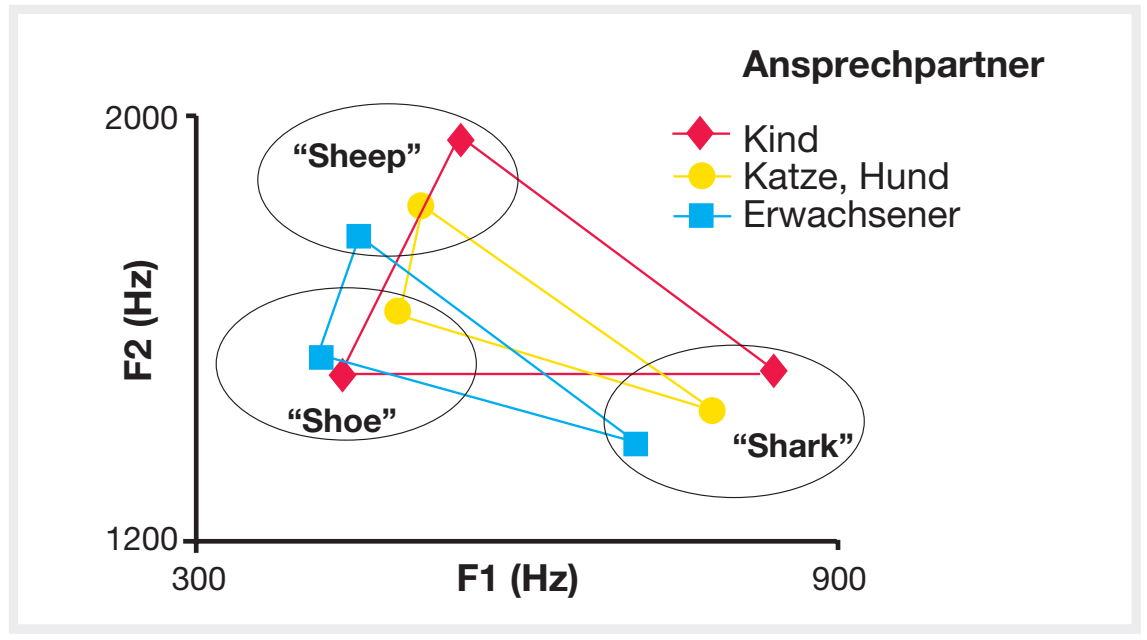

- Abb. 6 Mütter erhielten einen (Spielzeug-) Schuh und 2 Spielzeugtiere, einen Hai und ein Schaf, und sollten während ihrer Sprachaufnahme mit diesen Gegenständen operieren und damit die 3 Wörter mit im Englischen klar unterschiedenen Vokalen „i“, „a“ und „u“ („sheep“, „shark“, „shoe“) mehrfach aussprechen. Die Analyse der Frequenzen der ersten beiden Formanten zeigte erneut eine stärkere Unterscheidbarkeit (nach Daten aus [5, S. 1435).

ten sowie 14 Monaten sowohl aufgezeichnet, was zu bzw. mit dem Kind gesprochen wurde (dessen Sprachinput), als auch die sprachlichen Äußerungen des Kindes selbst (Sprachoutput). Die mit diesen Aufzeichnungen gewonnenen Daten (an das Kind gerichtete Äußerungen der Eltern sowie Äußerungen des Kindes) wurden in den jeweils etwa 45-minütigen Coaching-Sitzungen verwendet, um den Eltern detailliert darüber Rückmeldung zu geben, wie gut sie die Müttersprache beherrschten und wie oft sie diese einsetzten.

„Der Coach beschrieb, dass soziale Interaktionen, bei denen die Eltern in Müttersprache sprechen, eine reichhaltige, an das Kind gerichtete Sprache verwenden und das Kind in einen zusammenhängenden 1:1-Dialog verwickeln, der Sprachentwicklung des Kindes förderlich sind. Die Eltern wurden ermutigt, sich spezifische Situationen oder Aktivitäten auszudenken, die ihnen zu entsprechenden Interventionen Anlass geben. Beispielsweise wurden die Eltern gebeten, tägliche Routinen (wie Windelwechsel, Mahlzeiten, Badezeit) zu nennen, bei denen die Chance auf einen 1:1-Dialog in Müttersprache besonders groß ist, oder bei denen sie direkt zu ihrem Kind sprechen. Sie wurden dazu ermuntert, mit dem Coach zu diskutieren, warum diese speziellen Routinen für die Sprachentwicklung ihres Kindes wichtig sein könnten, und zudem Vorschläge zu machen, wie man über den Tag hinweg noch weitere ähnliche Momente schaffen könnte“, 5 beschreiben die Autoren ihr Vorgehen im Detail [9, S. e5].

5 „The coach described that social interactions in which parents speak in parentese, use rich language directed to the child, and engage the child in contingent, 1:1 back-and-forth exchanges may be beneficial to child language development. Parents were encouraged to think of specific scenarios or activities that help them engage in the intervention behaviors. For example, parents were asked to name examples of daily routines (such as diaper changes, meals, bath time) when they are particularly likely to engage their child in contingent 1:1 interactions, use parentese, or talk directly with their child. They were encouraged to discuss with the coach why these particular routines may be important for their child's language development, and propose what they can do to create additional, similar moments throughout the day." 
Der Coach spielte den Eltern zudem ausgewählte Audioaufnahmen vor und bat sie, auf bestimmte Details ihrer eigenen oder der kindlichen Äußerung zu achten. „Es wurden auch Beispiele für kindliches Plappern oder geäußerte Wörter vorgespielt, und die Eltern wurden ermutigt, zu erkennen, dass die ÄuBerungen von Babys oft sprachlich nicht vollständig sind. Die Eltern wurden darauf hingewiesen, dass es sich beispielsweise bei isolierten Vokalen wie ä oder a um Versuche des Kindes handeln kann, ein Wortzu sagen, insbesondere wenn die Äußerung von einem Fingerzeig oder einem deutlichen Blick auf einen Gegenstand begleitet wird" 6 [9, S. e5] Mit Hilfe spezieller Kärtchen wurden einzelne Beispielsituationen mit den Eltern durchgesprochen und an die Möglichkeiten zu Dialogen und Ansprachen in Müttersprache erinnert. „Schließlich besprach der Coach den nächsten zu erwartenden sprachlichen Meilenstein des Kindes (z. B. Lautwiederholungen, erste Wörter) und Strategien zur Unterstützung der Sprachentwicklung, indem das Kind in altersgerechte soziale Interaktionen eingebunden wird, d. h. das Interventionsverhalten (Dialoge der Eltern mit dem Kind, direktes Ansprechen des Kindes) beinhalten“7 $[9$, S. e6].

Zur Analyse der Tonaufnahmen wurde ein erheblicher Aufwand betrieben. Zwar lassen sich Wörter - für Kind und Erwachsene getrennt - automatisch mittels entsprechender Software zählen, die Art der Interaktion (z. B. Dialog in normaler Sprache oder in Müttersprache, durch Vater oder Mutter oder eine andere Person) wurde jedoch durch eigens hierfür trainierte Forschungsassistenten manuell kodiert (die

6 „Instances of the child's babbling or producing words were also played, and parents were encouraged to recognize that infants' utterances are often not fully formed. Parents were told that an isolated vowels such as/æ/or/ / are speech vocalizations that may reflect the child's attempt to produce a word, particularly when the utterance is accompanied by a finger point or consistent eye gaze to an object."

7 „Finally, the coach discussed the child's next expected language milestone (i. e., canonical babbling, first words) and strategies to support language growth, through engaging their child in age-appropriate social interactions that include the intervention behaviors (parentese, contingent 1:1 interactions, speaking directly with the child)."

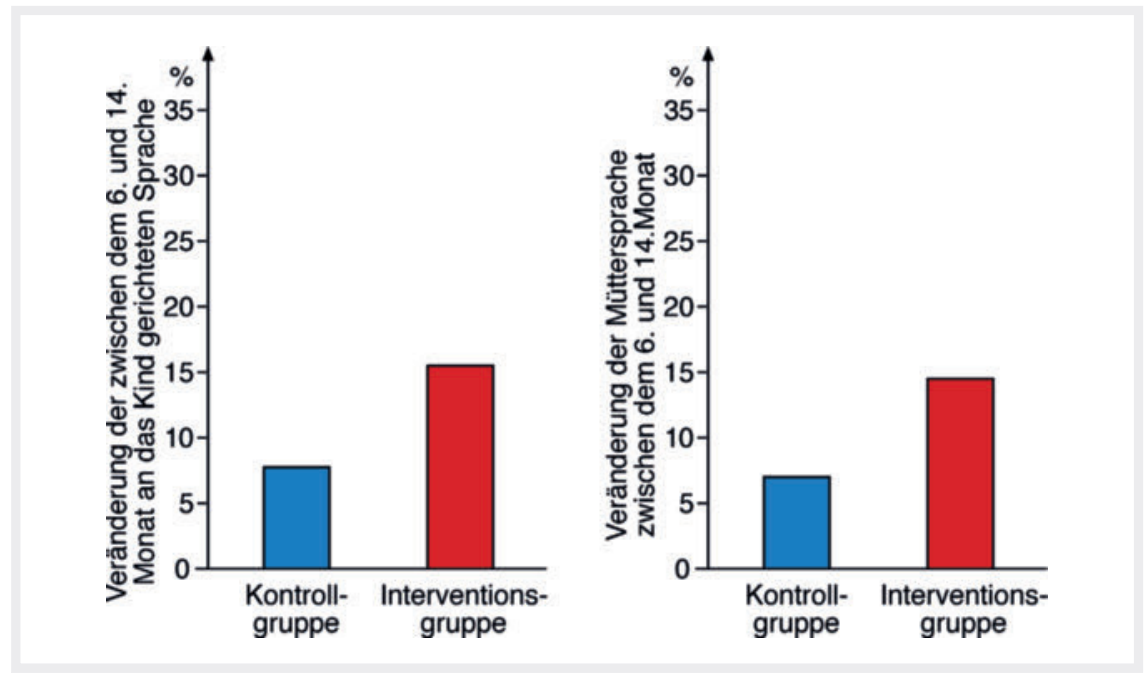

- Abb. 7 Prozentuale Veränderungen von an das Kind gerichteter Ansprache (links) und Verwendung von Müttersprache (rechts) zwischen dem 6. und 14. Monat in der Kontrollgruppe (blau) und der Interventionsgruppe (rot) (nach Daten aus [9, S. e10]).

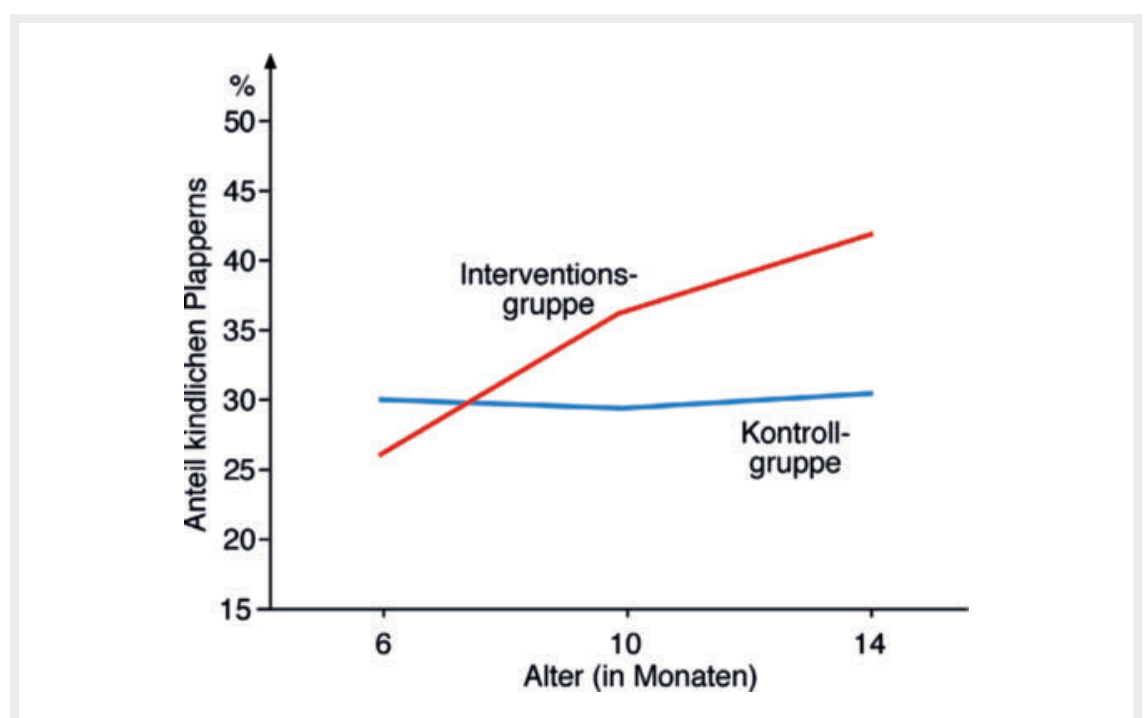

- Abb. 8 Kindliches Plappern in Prozent in der Kontrollgruppe (blau) und der Interventionsgruppe (rot) über die 3 Messzeitpunkte hinweg (nach Daten aus [9]).

Reliabilität zwischen den Forschungsassistenten lag für alle Variablen über $90 \%)$.

\section{Coaching in Müttersprache fördert die Sprachentwick- lung beim Kind}

Zu den Ergebnissen ist zunächst anzumerken, dass sich die Interventions- und Kontrollgruppe in den gemessenen Variablen der Sprachentwicklung zu Beginn der Studie unterschieden, und dass es einen Effekt der Variable „Messzeitpunkt“ (d. h. 6, 10 und 14 Monate) auf die meisten untersuch- ten Variablen gab. Mit anderen Worten: Bei den Kindern fand Sprachentwicklung statt und dies zeigte sich in den Messungen. Interessant waren die Wechselwirkungen zwischen den Variablen „Messzeitpunkt“ und „Gruppe“ (Kontrolle versus Intervention): Sowohl die an das Kind gerichtete Ansprache als auch die Verwendung von Müttersprache waren in der Interventionsgruppe signifikant stärker gestiegen. Diese Steigerungen waren sowohl zwischen 6 und 10 Monaten als auch zwischen 10 und 14 Monaten signifikant ( $\triangleright$ Abb. 7). Die Intervention bei den Eltern hatte messbare Auswirkungen auf die Sprachentwick- 

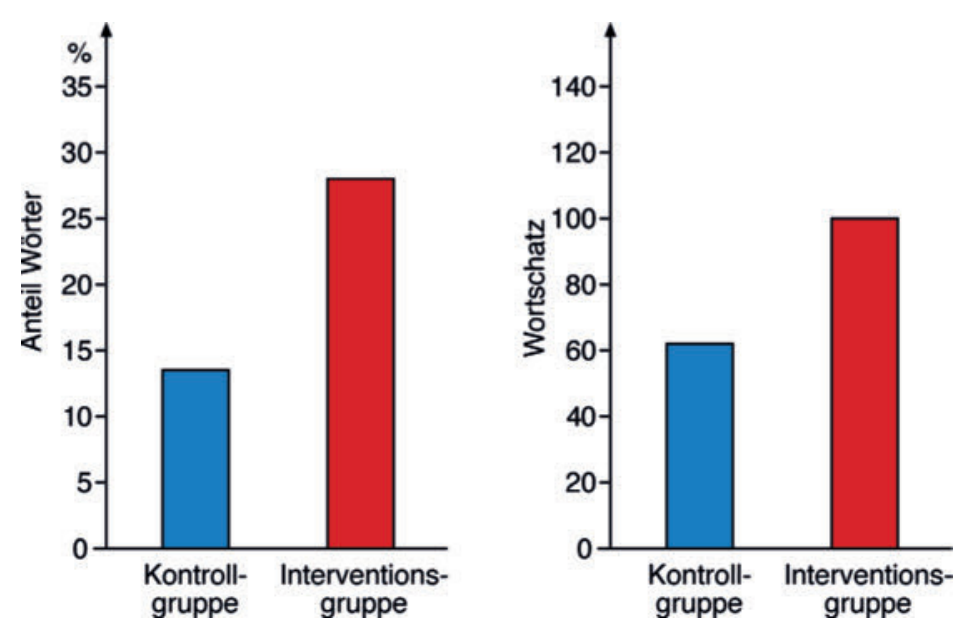

- Abb. 9 Sprachentwicklung bei Kindern im Alter von 18 Monaten, deren Mütter (von Monat 6 bis 14) ein Coaching in Müttersprache erhielten (Interventionsgruppe, $n=48$; rot) sowie bei Kindern einer Kontrollgruppe ( $n=23$; blau). Die linken Säulen geben den Prozentsatz der Wörter an allen lautlichen Äußerungen der Kinder an. Bei den rechten Säulen handelt es sich um die Ergebnisse des bekannten und reliablen Fragebogens für Eltern zur Sprachentwicklung von Kindern im Alter von 16 bis 30 Monaten, dem Communicative Development Inventory, Words and Sentences von MacArthur und Bates (CDI), mit dem der Wortschatz eines Kindes gemessen wird (nach Daten aus [10, S. e4]).

lung beim Kind. Wie aus $>$ Abb. 8 ersichtlich, nahm das kindliche Plappern über die 3 Messzeitpunkte hinweg nur in der Interventionsgruppe deutlich zu.

Eine kürzlich in elektronischer Form vorab publizierte Arbeit zur weiteren sprachlichen Entwicklung der Kinder zeigte, dass die Kinder der (elterlichen) Interventionsgruppe im Alter von 18 Monaten mehr Wörter pro Zeiteinheit sprachen und einen deutlich größeren Wortschatz besaßen als die Kinder der Kontrollgruppe ( $\mathbf{A b b . 9}$ ). Damit wurden erstmals die Auswirkungen auf die Sprachentwicklung von Babys nachgewiesen, wenn man Müttern die Müttersprache beibringt: „Coaching der Eltern steigert die sprachliche Entwicklung von Kindern und den wechselseitigen Dialog mit ihnen “, 8 lautet der Titel der Arbeit.

Die Autoren konnten mit ihrer weiteren Auswertung der Daten auch zeigen, dass das Training von Müttersprache unabhängig vom sozioökonomischem Status (SES) der Familien zu einer besseren Sprachentwicklung führt. Mit den Worten der Autoren: „Die hier vorgestellten Daten stützen die An-

8 „Parent coaching increases conversational turns and advances infant language development.“ sicht, dass eine experimentelle Verbesserung des elterlichen Sprach-Inputs über verschiedene SES-Hintergründe hinweg möglich ist. Wir zeigen, dass die Verbesserung der elterlichen sozialen Kommunikationsfähigkeiten sowohl robust als auch dauerhaft ist. Dies legt nahe, dass die Auswirkungen des elterlichen Coachings zwischen 6 und 18 Monaten sowohl Eltern als auch Kinder auf einen Pfad verbesserter sozialer Kommunikation bringen, was durch eine Zunahme wechselseitiger Dialoge in der Interventionsgruppe belegt wird. Die vorliegende Studie belegt damit den positiven sozialen Effekt von Müttersprache“9 [10, S. e4].

Die Autoren heben hervor, dass sie nur über gemessene Variablen berichten, die sie vor Beginn der Studie bereits festgelegt hat-

9 „The data presented here further support the notion that experimental manipulation of parental language input is possible, across a range of SES backgrounds. We show that enhancements in parental social communication skills are both robust and durable, and suggest that the effects of parent coaching between 6 and 18 months put both parents and children on a trajectory of enhanced social communication, evidenced by an increase in conversational turn-taking that we measured in the I group. As such, we argue that the present study presents evidence for a social benefit of parentese." ten. Das schließt natürlich nicht aus, dass „Müttersprache“ noch andere positive Auswirkungen hatte, die nicht gemessen wurden. Über die späteren Auswirkungen der Intervention können die Autoren (noch) nichts sagen, man darf jedoch auf weitere Publikationen gespannt sein. Sie geben jedoch zu bedenken, dass man nach allem, was man mittlerweile über die Bedeutung der kindlichen Sprachentwicklung für dessen weitere kognitive Entwicklung bis hin zum weiteren Bildungsverlauf und Lebensverlauf weiß, positive Effekte erwarten darf. Im Hinblick auf die Intervention heben sie vor allem hervor, dass den Eltern dadurch bewusst gemacht wurde, was sie sowieso tun, und dass durch diese erhöhte Bewusstheit ihres sprachlichen Umgangs mit ihren Kindern dieser Umgang qualitativ verbessert wurde.

„Obwohl alle Familien in der vorliegenden Stichprobe bereits zu Beginn der Studie Müttersprache verwendeten, zeigte sich in den Gesprächen während der Coaching-Termine, dass die große Mehrheit der Eltern sich der positiven Auswirkungen auf die Sprachentwicklung der Kinder nicht bewusst war, dass sie sich schwer damit taten, anzugeben, wann genau am Tag sie Müttersprache verwenden, und oft überrascht waren, wenn sie ihre Stimme in den Aufnahmen hörten. Unser Ansatz bestand darin, ihr Bewusstsein zu schärfen, indem wir die Forschungsergebnisse zu Müttersprache und anderen sozialen Aspekten der Eltern-Kind-Beziehung hervorheben und den Eltern durch das Anhören ihrer eigenen Aufnahmen zeigen, dass sie mit ihrem Kind auf eine besondere Weise umgehen können, um die Sprachentwicklung zu förern" 10 [10, S. e4]. Durch das Coaching, also

10 „Although all families in the present sample already used parentese at baseline, conversation during the coaching appointments revealed that the vast majority of parents were unaware of its positive effects on children's language growth, struggled to name specific moments of the day when they use parentese, and were often surprised to hear themselves using it in the recordings. Our approach was to raise their awareness by highlighting the research behind parentese and other social aspects of parent-child interactions, and by demonstrating to parents, through listening to their own recordings, that they can interact with their infant in ways that promote language growth." 

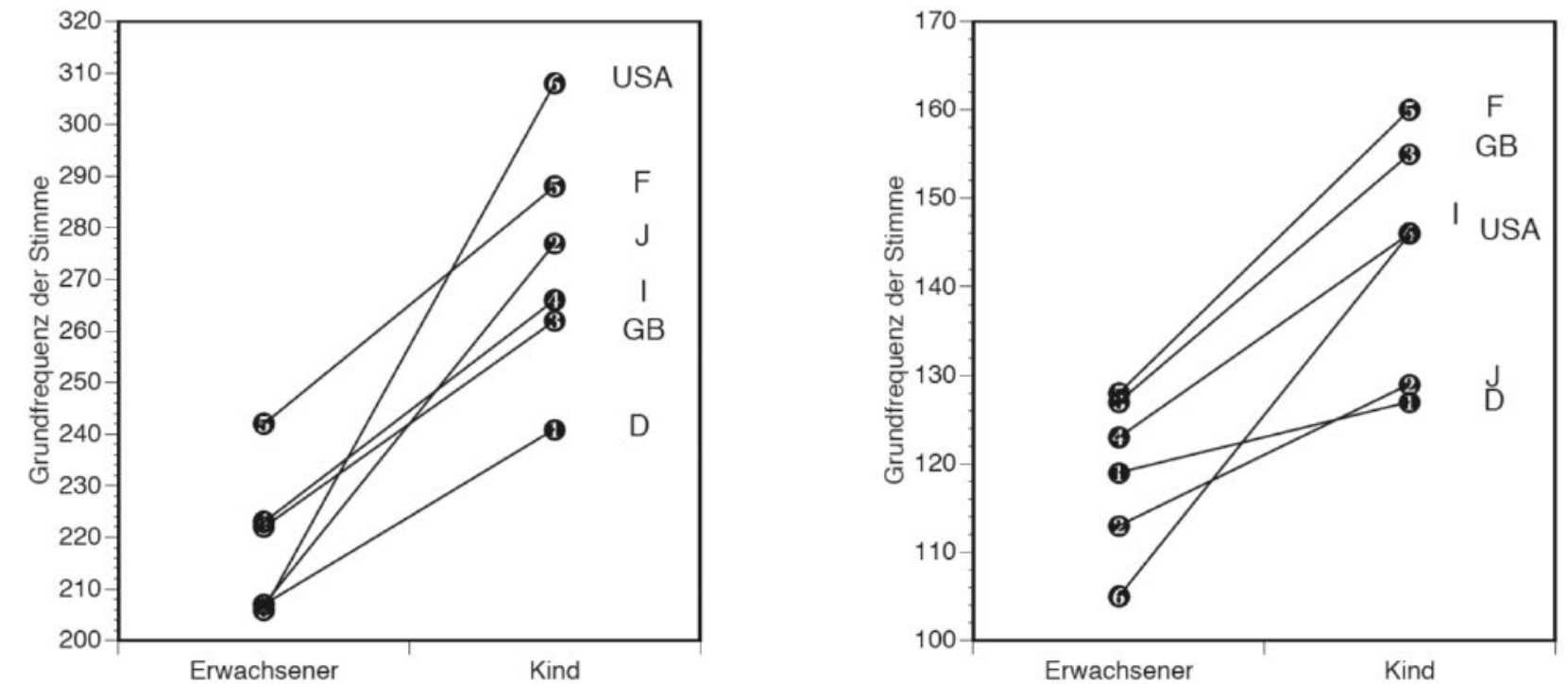

- Abb.10 Vergleich der Grundfrequenz des Sprechens von Frauen (links) und Männern (rechts) zu anderen Erwachsenen (jeweils links in der Grafik) und zu Kindern (jeweils rechts in der Grafik) in verschiedenen Sprachgemeinschaften (Deutschland, Frankreich, Großbritannien, Italien, Japan, USA). Wie man sieht wird überall von Frauen wie auch von Männern zu Kindern mit einer höheren Grundfrequenz gesprochen (nach Daten aus [10, S. 499]).

das Besprechen einzelner sprachlicher ÄuBerungen zu ihrem Kind und der Dialoge mit ihrem Kind, wurde den Eltern verdeutlicht, wie sie selbst jeden Tag ihr Sprechen variieren, den jeweiligen Alltagssituationen anpassen und damit bereichern können. Auch ganz konkrete kleine Sprachtipps wurden gegeben. Das kam bei den Eltern offenbar gut an: „Meistens verließen die Eltern die Coaching-Sitzungen dankbar und mit neuen Ideen, die sie zu Hause umsetzen konnten"11 [10, S. e4f]. Interessanterweise wurde so etwas wie "Müttersprache“ auch bei gehörlosen Müttern gefunden, die mit ihren Kindern in Gebärdensprache kommunizieren. Die dabei verwendete Gestik und Mimik zeigen ähnliche Veränderungen der Gebärden (größere Unterschiede durch "Übertreibung“, kürzere Sätze und längere Pausen zwischen den Wörtern) wie sie lautsprachlich gefunden wurden [33].

Müttersprache findet man bei Frauen in den verschiedensten Sprachgemeinschaften, wenn auch in unterschiedlicher Ausprägung. Vergleicht man die Höhe der Grundfrequenz der Stimme beim Sprechen mit Erwachsenen mit der Grundfrequenz beim Sprechen mit Kindern, so zeigt sich, dass

11 „Parents were generally grateful to leave the appointments with new ideas to implement at home." bei Engländerinnen und Deutschen die Stimme beim Sprechen mit Babys längst nicht so stark in die Höhe geht, wie dies bei den Amerikanerinnen der Fall ist [11] ( Abb. 10, links).

Bei den Vätern ist Müttersprache ebenfalls vorhanden, wird aber nicht so häufig verwendet. Eine andere Studie hingegen konnte keine Veränderung der Grundfrequenz bei Vätern, die zu ihren Kindern sprechen, finden [44]. Wahrscheinlich hängt es auch davon ab, wo man misst: Wie in der

- Abb. 10 (rechts) erkennbar, steigt die Grundfrequenz bei Vätern in den USA am stärksten an, am geringsten ist der Anstieg bei deutschen Männern (Gruppe mit dem geringsten Effekt einer Steigerung der Grundfrequenz beim Sprechen mit Babys um durchschnittlich gerade einmal $8 \mathrm{~Hz}$ ). Dass mir das Gequietsche von Amerikanerinnen (Gruppe mit dem größten Effekt von $102 \mathrm{~Hz}$ Frequenzsteigerung beim Sprechen mit Babys) aus vielfacher eigener Erfahrung heftig in nicht gerade positiver Erinnerung ist, liegt also vielleicht ganz einfach daran, dass ich ein deutscher Mann bin.

\section{Coaching für Väter?}

Sollten also - vor allem gerade wir deutschen - Väter flächendeckend Coaching in Müttersprache bekommen, damit der deut- sche Nachwuchs künftig - vor allem natürlich im internationalen Vergleich - sprachlich wieder eine Chance hat? An dieser Stelle muss ich die Freunde schneller einfacher Lösungen um Geduld bitten, und meine Arbeit mit dem Standard-Spruch „more research is needed" 12 beenden. - Warum?

Wenn auch noch nicht sehr viele, so gibt es tatsächlich schon ein paar Studien zu Vätern, die von der Entwicklungspsychologie erst Jahrzehnte nach den Müttern als Forschungsobjekt in den Blick genommen wurden. Mittlerweile ist der systematische Grund dieser historischen Tatsache bekannt: Beim Reden mit Babys haben die Frauen die Nase um das 2- bis 3-Fache gegenüber den Männern vorn und zwei Drittel bis drei Viertel des an Babys gerichteten Sprechens kommt von den Müttern [3]. Je älter das Kind jedoch wird, desto mehr nähern sich die Väter den Müttern an, was ihren Sprachinput für das Kind anbelangt. Väter sprechen also vor allem mit Babys eher wenig, mit 2-Jährigen schon eher und mit noch älteren Kindern noch mehr [22]. Dennoch ist es nicht so, dass der Vater keinen Einfluss auf die Sprachentwicklung seiner Kinder hat - im Gegenteil: Eine ganze Reihe von Untersuchungen konnte mitt-

12 Zu Deutsch etwa: „erst weitere Forschungsarbeiten werden ... Klarheit bringen...“ 
lerweile zeigen, dass der Vater einen über den Effekt der Mutter hinausgehenden und von der Mutter unabhängigen positiven Effekt auf die Sprachentwicklung und auch auf die soziale und emotionale Entwicklung der Kinder hat, die sich im Alter von 2, 3 und 5 Jahren der Kinder nachweisen lassen $[6,7]$.

Man weiß, dass Fragen (durch die Eltern) die Sprachentwicklung beim Kind fördern, insbesondere die im Englischen als „Wh-questions“ bezeichnete Fragen (Wann, Wo, Wer, Was). ${ }^{13}$ Solche Fragen sowie Nachfragen bei Unklarheiten kommen bei Vätern häufiger vor als bei Müttern, wenn sie mit ihren Kindern reden [21, 22, 35]. Auch verwenden Väter seltene Wörter häufiger, was zur Entwicklung des Wortschatzes der Kinder beiträgt. Wie eine Studie von Rowe und Mitarbeitern an 41 afroamerikanischen Vätern von 24 Monate alten Kindern zeigen konnte, kommen solche Fragen bei Vätern häufiger vor als bei Müttern und haben einen positiven Effekt auf die Sprachentwicklung, der sich bei den Kindern im Alter von 24 Monaten und 36 Monaten zeigt [36].

„Zusammenfassend lässt sich sagen, dass Väter für Kleinkinder besonders herausfordernde Kommunikationspartner darstellen, da sie eine große Anzahl von Äußerungen machen, die den Dialog fördern. Diese Äußerungen, insbesondere Wh-Fragen, entlocken den Kindern in der Tat komplexe Gespräche und haben positive Auswirkungen auf den Wortschatz und das spätere sprachgebundene Denken der Kinder" [35, S. 176]. ${ }^{14}$ Hierzu passt auch der Befund, dass Kinder von Vätern, deren an das Kind gerichtete Äußerungen weniger Wiederholungen enthalten, einen größe-

13 So schreiben beispielsweise Rowe und Mitarbeiter [37, S. 164]: „[...] there is a consistent and growing body of evidence that parent use of, particularly wh-questions, is positively associated with children's vocabularyskills."

14 "In sum, fathers are challenging communicative partners for toddlers in that they pose a large number of conversational-eliciting utterances. These utterances, particularly wh-questions, do indeed elicit complex talk from children and are predictive of children's concurrent vocabulary and later verbal reasoning skills." ren Wortschatz im Alter von 2 Jahren aufwiesen [39].

Nach der Bridge-Hypothese [43] sprechen Väter nicht so wie Mütter, allerdings auch nicht ganz so wie Fremde, sondern bilden sozusagen eine Brücke für die Kinder von der (gewohnten, unterstützenden, „quietschenden “) Mutter zu irgendwelchen emotional und attentional eher zurückhaltenden völlig fremden Menschen, mit denen ein Kind ja auch sprachlich umgehen lernen muss. Väter stehen damit zwischen der im Haus und der außer Haus (öffentlich) gebräuchlichen Sprache und bilden somit auch eine sprachliche Brücke zum weitergehenden sozialen Umfeld des Kindes. Immer wieder findet sich in der Literatur ganz allgemein der Hinweis, dass Väter einen positiven Einfluss auf die sprachliche Entwicklung haben, wenn 2 Voraussetzungen gegeben sind: Sie sind nicht ungebildet und nicht arm. Anders gewendet: Ein Vater, der seine Familie kaum ernähren kann und wenig gebildet ist (nur High-school-Abschluss oder weniger), wird die Entwicklung des Kindes eher ungünstig beeinflussen. ${ }^{15}$

\section{Zusammenfassende Diskus- sion}

Man weiß schon lange, dass die Verarbeitung von Sprachinput einen sehr großen Einfluss auf die sprachliche Entwicklung eines Kindes und damit auf dessen Bildungskarriere hat $[30,42]$. Dies gilt sowohl quantitativ (die Anzahl der gehörten Wörter bei Schuleintritt sagt die sprachlichen Fähigkeiten in der Schule und sogar den letztlich erreichten Bildungserfolg voraus) $[16,17]$ als auch qualitativ (die Güte und Tiefe der Dialoge haben einen deutlichen moderierenden, über die reine Anzahl der Wörter hinausgehenden Einfluss) [34, 35]. Zudem weiß man um die Abhängigkeit des Sprechens in Familien von deren Bildungsniveau: Je gebildeter Menschen sind, desto mehr reden sie miteinander. Weil Bildung auf Sprache aufbaut („Unterricht“ ist sprachlich vermittelte Bildung) und der Wortschatz und die Wortgewandtheit eines Kindes abhängig ist von seiner

15 Die Auswirkungen des sozioökonomischen Status der Familie auf die Entwicklung der Kinder sind dramatisch und vielfach nachgewiesen. frühen Sprachentwicklung in der Babyzeit bis zu 2 Jahren, kommt dem frühen Sprechen eine große Bedeutung zu.

Die hier vorgestellten Studien zeigen, dass Kinder noch nicht so gut hören, wenn ihr Spracherwerb schon erfolgt. Das Resultat ist, dass Mütter ihre Sprache ändern, damit beim Kind mehr ankommt: Sie sprechen mit höherer Stimme und stärker wechselnder Tonhöhe, machen längere Pausen und sprechen in kürzeren Sätzen. Diese Müttersprache findet man in verschiedenen Sprachgemeinschaften, wenn auch in unterschiedlicher Ausprägung (und sogar bei stummen Müttern, die in Gebärdensprache analoge Veränderungen produzieren). Sie wirkt sich erwiesenermaßen positiv auf die sprachliche Entwicklung von Kindern (übrigens bei beiden Geschlechtern gleichermaßen) aus. Macht man Mütter von 6 bis 10 Monate alten Kindern auf die Besonderheiten und die Bedeutung ihrer eigenen müttersprachlichen Äußerungen in wöchentlichen Coaching-Sitzungen mit praktischem Feedback zu daheim aufgenommenen Sprachaufnahmen aufmerksam, so führt dieses Training zu einer besseren Sprachentwicklung bei den Kindern, wie Tests der Kinder im Alter von 14 und 18 Monaten zeigen konnten.

Was die Väter anbelangt, so leisten auch sie - sofern sie einigermaßen gebildet sind und für das materielle Wohl der Familie aufkommen können - einen eigenen Beitrag zur Entwicklung ihrer Kinder, auch im Hinblick auf die Sprachentwicklung. Sie stellen mehr Fragen und klären mehr Ungenauigkeiten im Dialog mit den Kindern auf. Während Mütter noch bis ins 3. Lebensjahr des Kindes hinein zunehmend mehr Müttersprachanteil an allen ihren Äußerungen dem Kind gegenüber aufweisen, ist der Anteil und der Ausprägungsgrad der Müttersprache bei Vätern (insbesondere hierzulande) von Anfang an geringer und nimmt im Laufe der Zeit ab, sodass Väter sprachlich eine Brücke vom Zuhause in die „Welt draußen“"schlagen, denn sie sprechen schon mit kleinen Kindern (ab 2 Jahren) ähnlich wie Fremde, was sich wahrscheinlich förderlich auf die Sprachentwicklung der Kinder auswirkt.

Für Empfehlungen zu einem Coaching der Väter in Müttersprache reichen die vor- 
liegenden Daten noch nicht aus. Die Forschung ist noch nicht so weit, um die unterschiedlichen, jeweils für Väter und Mütter spezifischen sprachlichen Anpassungen ihrer an das Kind gerichteten Sprache abschließend beurteilen zu können. „Mehr Müttersprache (als bislang) bei Kindern unter einem Jahr, weniger (als bislang) bei Kindern über 2 Jahren “ - so könnte eine Empfehlung lauten, wenn man noch mehr wüsste. Die positiven Auswirkungen des Bewusstmachens des sprachlichen Umgangs mit dem eigenen Kind, die vor allem (aber nicht nur) bei Müttern gefunden wurden, dürften auch Vätern dabei helfen, ihren sprachlichen Umgang mit den Kindern zu optimieren. Ob sie hierzu jedoch in Müttersprache gecoacht werden müssen, wissen wir (noch) nicht.

Interessenkonflikt

Es besteht kein Interessenkonflikt.

\section{Korrespondenzadresse}

\section{Prof. Dr. Dr. Manfred Spitzer}

Universität Ulm

Abteilung für Psychiatrie

Leimgrubenweg 12-14

89075 Ulm

\section{Literatur}

[1] Bahrick LE, McNew ME, Pruden SM, et al. Intersensory redundancy promotes infant detection of prosody in infant-directed speech. J Exp Child Psychol 2019; 183: 295-309

[2] Beals D, Tabors P. Arboretum bureaucratic and carbohydrates: Preschoolers' exposure to rare vocabulary at home. First Lang 1995; 15(43 Pt 1): 57-76

[3] Bergelson E, Casillas M, Soderstrom M, et al. What Do North American Babies Hear? A large-scale cross-corpus analysis. Dev Sci 2019; 22: e12724. doi: 10.1111/ desc. 12724

[4] Bregman AS. Auditory scene analysis: The perceptual organization of sound. Cambridge: MIT Press 1990

[5] Burnham D, Kitamura C, Vollmer-Conna U. What's New, Pussycat? On Talking to Babies and Animals. Science 2002; 296: 1435
[6] Cabrera NJ, Shannon JD, Tamis-LeMonda C. Fathers' influence on their children's cognitive and emotional development: From toddlers to pre-K. Applied Development Science 2007; 11: 208-213

[7] Cabrera NJ, Fitzgerald HE, Bradley RH, et al. The ecology of father-child relationships: An expanded model. Journal of Family Theory and Review 2014; 6: 336-354

[8] Ferguson B1, Lew-Williams C. Communicative signals support abstract rule learning by 7-month-old infants. Sci Rep 2016; 6 : 25434. doi: 10.1038/srep25434

[9] Ferjan Ramírez N, Lytle SR, Fish M, et al. Parent coaching at 6 and 10 months improves language outcomes at 14 months: A randomized controlled trial. Dev Sci 2019; 22: e12762

[10] Ferjan Ramírez N, Roseberry Lytle S, et al. Parent coaching increases conversational turns and advances infant language development. PNAS 2020; 117: 3484-3491. doi/10.1073/pnas.1921653117

[11] Fernald A. Four-month-old infants prefer to listen to motherese. Infant Behavior and Development 1985; 8: 181-195

[12] Fernald A. Intonation and communicative intent in mothers speech to infants: is the melody the message? Child Development 1989; 60: 1497-1510

[13] Fernald A. Prosody in speech to children: prelinguistic and linguistic functions. Annals of Child Development 1991; 8: 43-80

[14] Fernald A. Approval and disapproval: infant responsiveness to vocal affect in familiar and unfamiliar languages. Child Development 1993; 64: 657-674

[15] Golinkoff RM, Hoff E, Rowe ML, et al. Language matters: Denying the existence of the 30-million-word gap has serious consequences. Child Dev 2019; 90: 985-992

[16] Hart B, Risley TR. Meaningful differences in the everyday experience of young american children. Baltimore, MD: PH Brookes 1995

[17] Kornilov SA, Zhukova MA, Ovchinnikova IV, et al. Language Outcomes in Adults with a History of Institutionalization: Behavioral and Neurophysiological Characterization. Sci Rep 1991; 9: 4252. doi: 10.1038/ s41598-019-40007-9

[18] Kuhl PK, Andruski JE, Chistovich IA, et al. Cross-Language Analysis of Phonetic Units in Language Addressed to Infants. Science 1997; 277: 684-686

[19] Kuhl PK, Ramírez RR, Bosseler A, et al. Infants' brain responses to speech suggest analysis by synthesis. PNAS 2014; 111: 11238-11245

[20] Laing CE, Bergelson E. Mothers' Work Status and 17-month-olds' Productive Vocabulary. Infancy 2019; 24: 101-109

[21] Leaper C, Anderson KJ, Sanders P. Moderators of gender effects on parents' talk to their children: A meta-analysis. Dev Psychol 1998; 34: 3-27

[22] Leech KA, Salo VC, Rowe ML, et al. Father input and child vocabulary development: the importance of Wh questions and clarification requests. Semin Speech Lang 2013; 34: $249-259$

[23] Liu H-M, Kuhl PK, Tsao F-M. An association between mothers' speech clarity and infants' speech discrimination skills. Developmental Science2003; 6: F1-F10

[24] Lordier L, Meskaldji DE, Grouiller F, et al. Music in premature infants enhances high-level cognitive brain networks. PNAS 2019; 116: 12103-12108

[25] Ma W, Golinkoff RM, Houston D, et al. Word Learning in Infant- and Adult-Directed Speech. Lang Learn Dev 2011; 7: 185-201

[26] Marcus GF, Vijayan S, Rao SB, et al. Rule Learning by Seven-Month-Old Infants. Science 1999; 283: 77-80

[27] Merz EC, Maskus EA, Melvin SA, et al. Socioeconomic Disparities in Language Input Are Associated With Children's Language-Related Brain Structure and Reading Skills. Child Dev 2019 Mar 28. doi: 10.1111/ cdev. 13239

[28] Morningstar M, Garcia D, Dirks MA, et al. Changes in parental prosody mediate effect of parent-training intervention on infant language production. J Consult Clin Psychol 2019; 87: 313-318

[29] Owens RE. Language Development. 9. Auflage. Boston: Pearson 2016

[30] Pace A, Alper R, Burchinal MR, et al. Measuring success: Within and cross-domain predictors of academic and social trajectories in elementary school. Early Childhood Research Quarterly 2019; 46: 112-125

[31] Ramírez-Esparza N, García-Sierra A, Kuhl PK. Look who's talking: speech style and social context in language input to infants are linked to concurrent and future speech development. Dev Sci 2014; 17: 880-891

[32] Ramírez-Esparza N, García-Sierra A, Kuhl PK. Look Who's Talking NOW! Parentese Speech, Social Context, and Language Development Across Time. Front Psychol 2017; 8: 1008. doi: $10.3389 /$ fpsyg.2017.01008

[33] Reilly JS, Bellugi U. Competition on the face: Affect and language in ASL motherese. J Child Lang 1996; 23: 219-239

[34] Romeo RR, Leonard JA, Robinson ST, et al. Beyond the 30-Million-Word Gap: Children's Conversational Exposure Is Associated With Language-Related Brain Function. Psychol Sci 2018; 29: 700-710

[35] Rowe ML, Coker D, Pan BA. A comparison of fathers' and mothers' talk to toddlers in low-income families. Soc Dev 2004; 13(2): 278-291

[36] Rowe ML. A longitudinal investigation of the role of quantity and quality of child-di- 
rected speech in vocabulary development. Child Dev 2012; 83: 1762-1774

[37] Rowe ML, Leech KA, Cabrera N. Going Beyond Input Quantity: Wh-Questions Matter for Toddlers' Language and Cognitive Development. Cogn Sci 2017; 41 Suppl 1: 162-179

[38] Schneider B, Trehub SE. Behavioural assessment of basic auditory abilities. In: Schneider B, Trehub SE (Hrsg). Auditory development in infancy. New York: Plenum Press, 1985, 101-114

[39] Schwab JF, Lew-Williams C. Language learning, socioeconomic status, and child-directed speech. Wiley Interdiscip Rev Cogn Sci 2016; 7: 264-275

[40] Schwab JF, Rowe ML, Cabrera N, et al. Fathers' repetition of words is coupled with children's vocabularies. J Exp Child Psychol 2018; 166: 437-450

[41] Spitzer M. Musik im Kopf, 2. Auflage. Stuttgart: Schattauer 2014

[42] Spitzer M. Am Anfang war das Wort. Nervenheilkunde 2015; 34: 466-468

[43] Tomasello M, Conti-Ramsden G, Ewert B. Young children's conversations with their mothers and fathers: Differences in breakdown and repair. J Child Lang 1990; 17: $115-130$

[44] Van Dam M, De Palma P, Strong WE. Fathers' use of fundamental frequency in motherese. Poster presented at the 169th Meeting of the Acoustical Society of America, Pittsburgh 2015

[45] Weber A, Fernald A, Diop Y. When Cultural Norms Discourage Talking to Babies: Effec- tiveness of a Parenting Program in Rural Senegal. Child Dev 2017; 88: 1513-1526

[46] Werner LA, Leibold L]. Auditory develop ment in children with normal hearning. In: Tharpe A (Hrsg). Comprehensive Handbook of Pediatric Audiology, 2. Auflage. San Diego: Plural Publishing 2016

Bibliografie

DOI https://doi.org/10.1055/a-1094-9512 Nervenheilkunde 2020; 39: 328-337

(c) Georg Thieme Verlag KG Stuttgart - New York ISSN 0722-1541 